\title{
A Strange Algorithm for Fingerprint Based Age Estimation using Frequency Domain and Pattern Recognition Techniques
}

\author{
T.Arulkumaran, V.Ganesan
}

\begin{abstract}
-identifying the age from the unique mark of a new individual is a developing field of constructing studies. to perform this age component diverse systems had been extensively applied. Dominant a part of strategies makes use of the parameters like facet check and area broadness of a given particular finger influence for identity of the age estimation of that distinct person. Unique mark exam has enormous potential as a functioning machine for recognizable evidence of an person depending on both medical just as on restorative parameters. on this exploration work to select the age along their relationship recurrence vicinity and example acknowledgment methods were actualized for a hit grouping of finger prints two Dimensional Discrete Wavelet's (DWT) alongside independent component evaluation (ICA) are utilized to gauge the age factor from the unique mark statistics base. The statistics base which contains round 500 fingerprints of humans involving extraordinary age bunches inside the scope of 10 to 60 is considered for our exploration work. Multi-modular aid Vector gadget primarily based classifier is utilized for particular mark grouping. The exploratory consequences were arranged and from alongside those strains were given plots it changed into discovered that the talent of the proposed method is good.
\end{abstract}

Keywords-Age guarantee, Discrete Wavelet transform technique, help Vector system Classifier, unbiased issue analysis, part examination

\section{INTRODUCTION}

Fingerprints are seemed as one of the most dependable Validation approach in respectable courtroom around the globe. Fingerprints is the most broadly applied and recognizable biometric parameter used by special places of work for verification and approval purposes. Fingerprints offers an assortment of statistics's which can be organized solely to both determine or look at or to settle on a preference dependent on the dataset. development of affirmation or distinguishing proof strategies are critical studies territories in protection based totally facts research. Plans of those kinds of essential techniques from biometric based totally fingerprints are elaborate yet they're extraordinarily powerful and dependable in the gift scenario.

A human unique finger impact is a portrayal of edges which are available on the upper raised layer of epidermis at the arms and $\mathrm{ft}[1-3]]$. the threshold examples are one of a type to everybody and no two human beings, even indistinguishable twins might not have equal examples (maltoni and cappelli, 2006)[7]. Fingerprints can be of either

Revised Manuscript Received on July 10, 2019.

T.Arulkumaran, Research Scholar, Dept. of ETCE, Sathyabama Institute of Science \& Technology, Chennai, Tamil Nadu, India. (Email:rajarul84@yahoo.co.in)

V.Ganesan, Assistant Professor, Dept. of ETCE Sathyabama Institute of Science \& Technology, Chennai, Tamil Nadu, India.(Email:vganesh1711@gmail.co.in) curve form or circles or of whorls. The examples are reliable and everlasting for the entire existence expectancy of that character.light meditated shape Optical Scanners over these examples are stuck via rate coupled devices and modified over into electrical data .these statistics's are the portrayal of the threshold examples present in the palms. in preference to optical sensors capacitive sensors are additionally now after which utilized (galton, 1982).

Automated precise mark calculations are utilized to recognize the examples of edges and valley's[4].those calculations are useful in computer safety and human distinguishing proof. Anthropologists likewise use these kind of calculations to pick out the a while structure the fingerprints separated from the artefact's. facet broadness' contrasts typically amongst male and women of identical age gatherings. the edge tally likewise contrasts among guys and girls. Epidermal edges plan offers an collection of houses that imitate the anatomical and organic shape of someone. Dermatoglyphics or the research of precise mark edges includes measurably range among the diverse genders and ethnic gatherings.

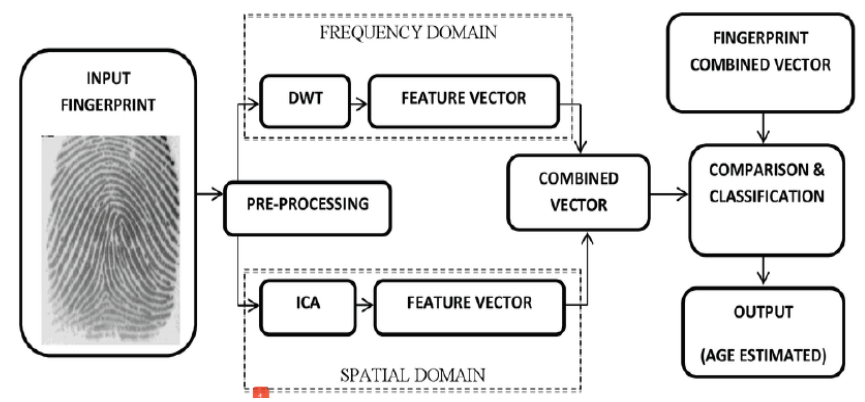

Fig.1. Generalized Block for Age Estimation

The obtained fingerprints are taken as real time. The finger prints taken for age estimation machine constitutes of digital finger print pictures as its enter that is then it is going to be converted. The algorithm may be advanced using Matrix Laboratory (MATLAB) software. we've got use two Dimensional Discrete Wavelet's (DWT) along with impartial component analysis (ICA) [1] are used to estimate the age component from the finger print facts base. The acquired finger prints go through to pre-processing operation for enhancement and getting rid of the undesirable noise and distorted portions. sooner or later after pre-processing level 


\section{A Strange Algorithm For Fingerprint Based Age Estimation Using Frequency Domain And Pattern Recognition Techniques}

the finger print undergoes to 2 degrees of feature vector extraction, one degree is frequency domain function vector obtaining via present process the discrete wavelet decomposition and the second degree is obtaining spatial stage feature vector present process thru impartial element evaluation(ICA). Then the both the frequency power vector values and spatial feature vector values are combine and that is given to the multi elegance SVM classifier which classifies the finger prints as to which magnificence it belongs.

\section{RELATED WORKS}

Human fingerprints had been determined on a giant quantity of archeological things and true items. no matter the fact that these revelations offer signal to the exhibit that an antiquated people conscious of the individuality of the fingerprints, it became not till the past due sixteenth century that the current logical precise mark techniques had been first began (Jain et al 2003)[8].In 1686 ,a life structures researcher and instructor on the university of Bologna, Marcelloo Malpighi referred to in his works in the presence of edges, circles and Spirals in fingerprints [9].

Specific mark acknowledgment is is based upon vital houses: (I) ingenuity: the essential fingerprints highlights don't fluctuate with time; and (ii) distinction: the examples of finger influence are one of a type to each awesome character. The legitimacy of the ingenuity has been constructed up via the life structures and morphogenesis of erosion facet pores and skin. even as the following purpose has been frequently stated to be genuine depending on trial outcomes, the primary logical premise of precise mark distinction has no longer been authoritatively tried. for this reason, specific finger influence verification is currently being tested in lots of courtroom cases. We address the problem of specific mark independence via identifying the degree of data available in particulars focuses to find out a correspondence amongst two specific finger impact photographs. We infer an articulation which assesses the chance of inaccurately corresponding details primarily based showings from two abnormal fingerprints. receive that the likelihood of a unique finger impression with 36 details purposes of the fingerprints will impart 12 details focuses to another haphazardly picked particular finger influence with 36 info functions of the fingerprints is 6:10 and 10:eight. those capacity effects assessed traits are likened with everyday unique finger influence matcher accuracy effects. Our outcomes exhibit that (I) the presentation of programmed unique finger affect matcher does now not approach the hypothetical execution, (ii) in opposition to the usual view particular finger impression coordinating isn't truthful and prompts a few misguided affiliations, and (iii) because of the confined facts substance of the details based totally portrayal, the programmed framework fashioners ought to find the utilization of non-particulars primarily based data gift in the fingerprints.

The character of the human fingerprints is broke down a good way to specific their Age and Gender and connection of Ridge be counted and Ridge thickness to Valley thickness share (RTVTR) [5] on sex identification, the research of precise finger impact is to examine the viability of physical biometrics (thumb print) so that you can distinguish the age and sexual orientation in character humans. An software framework was considered to trap the fingerprints of tried population over a completely unique mark biometric scanner interfaced to the pc through accepted Serial Bus (USB) and positioned away in Microsoft square Server database, even as back-unfold neural gadget method can be applied to prepare the put away finger affect. The precise points of this examination are to: by means of utilising the precise finger impact biometric scanner to gather various unique finger impression from people together with their age and sexual orientation. To broaden a model and enhance a completely unique finger influence based distinguishing evidence framework to finish up age and sexual orientation of human beings and to assess the set up framework [8].

On creative objects from stylish earthenware workshops, epidermal area expansiveness of human fingerprints turned into inspected [9]. Our exam has proven that suggest epidermal side broadness (MRB) as noticed on pottery may be applied as an age pointer (from delivery to improvement) and sex of the antiquity maker in improvement. in this examination, they endorse some other method for filtering, estimating and records taking care of.

The fine approach for age estimation (utilizing the equation proposed by using Kamp et al. adjusted after decrease through 7.five\%) produced outcomes with imply blunder of assessments -0.18 years ( $\mathrm{SD}=2.36$ years), center preferrred mistake of appraisals was 1.71 years and the outright mistakes were higher than five years simply in $3.6 \%$ of cases. on this manner in a selected ethnic accumulating epidermal edge broadness of fingerprints on pottery is suitable for searching at of man or woman's ages [10]. the amount of fingerprints and edges consistent with character had no effect on blunders in estimation. In adults, sexual dimorphism unmistakably existed notwithstanding the truth that antiques had been produced the use of various sorts of fired dirts. edge broadness is $9 \%$ extra outstanding in men contrasting and women [11]. In general, MRB underneath zero. $39 \mathrm{~mm}$ infers a sub-grown-up person underneath 15 years vintage and MRB values above $0.52 \mathrm{~mm}$ come just from grown-up men. though, age changes of facet broadness in youngsters cover with grown-up sexual dimorphism and eventually if there have to stand up an prevalence of MRB values from zero. 39 to 0 .fifty two mm, irregularity in both age and sex must be considered. on the off threat that this approach is moreover grown, best the subjective highlights of fingerprints and residences of earthenware mud, it has great capacity for illuminating the social basis of pottery making in antique human societies.

We propose a unique methodology programming based multi-biometric technique of age estimation which expects to find out the age of the person by means of utilising highlight extraction and arrangement. The given info photograph before everything reviews the pre-managing systems like difference development, histogram adjustment, binarization, and so on. similarly the element vector became eliminated from the picture using Discrete Wavelet transform (DWT) approach and independent issue evaluation (ICA) device [8].

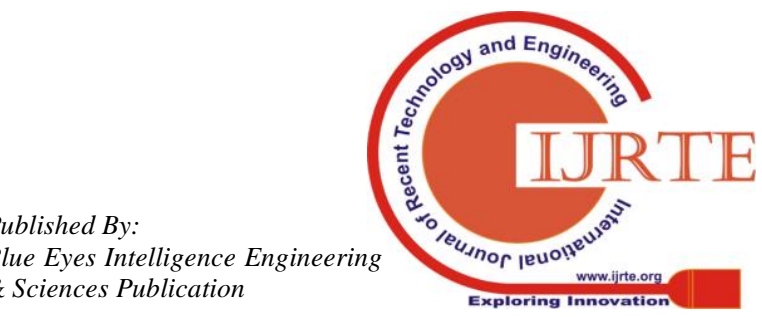


We utilize a classifier known as Multi-elegance SVM structures for the association dependent on preparing informational index given to that. Programming based totally system being blanketed furthermore, it exhibits the same old factors of interest of this form of methodologies: short, as it just desires one photo (i.e., a comparable particular mark test is gained for biometric acknowledgment) to recognize the age of the humans; non-nosy; clean to use (straightforward to the consumer); shabby and easy to settle in formally sensible frameworks (as no new bit of equipment is required). an extra little bit of leeway of this proposed strategy is its pace and its low unpredictability, which makes it very appropriate to paintings on proper conditions (one in every of the right traits of this kind of techniques). because it does not ship any feature explicit property (e.g., info focuses, iris position or face identity), the calculation burden required for image making ready reasons for current is faded making use of general photo nice estimates short to determine, joined with primary classifier. it has been experienced on broadly reachable databases of specific mark, wherein it has arrived at effects completely practically equal to the ones were given on the equivalent trial method by means of step by step complex characteristic specific pinnacle-located techniques from the best in class

\section{A. PRE-PROCESSING:}

The initial step of particular mark research is photo improve bureaucracy it consist of an accumulation of methods that try and improve the visible appearance of an any specific finger influence image or to make an interpretation of the picture to a structure appropriate for concentrate with the aid of a human or a component. The most and giant Enhancement techniques like differentiation development, histogram leveling, binarization and reversing are utilized consistent with the want of the unique finger impression photo to be upgraded. The particular finger influence photo is resized into $512 \times 512$ frameworks sizes. The received precise finger affect photo stories to photo improve improves the nature of the edges and valleys of finger affect picture. The data photo that's in dim scale worth is modified over into double well worth. The yield of particular mark pre-making ready is appeared in Fig 2. After pre-

dealing with, the fingerprints enjoy further tactics. improvement strategies modifications from particular finger influence from one individual to precise finger affect from someone else and furthermore for various databases. Gotten poor nice fingerprints can be more suitable for the development of the methods.
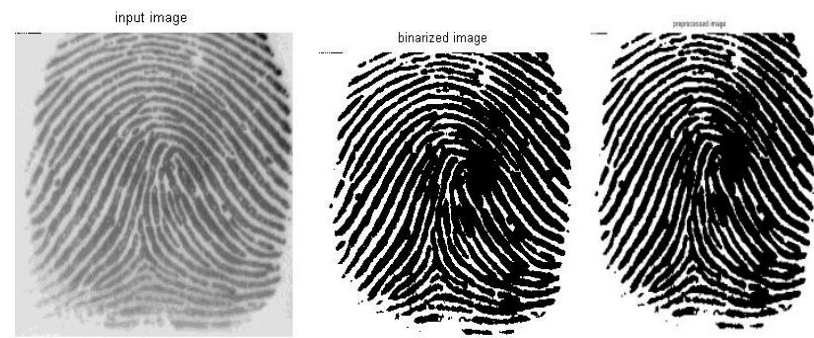

Fig. 2. Pre-processed finger print Image
The particular mark image studies discrete wavelet alternate after pre-getting ready for buying the element vector. Discrete Wavelet changes have been utilized every from time to time in photo managing for highlight vector extraction, age, de-noising, stress and picture fantastic-dreams. two dimensional Discrete Wavelet transform (DWT) breaks down the given particular mark picture into numerous sub-agencies which can be restricted in recurrence and course. The various frequencies vitality vectors received from photo disintegration permits the isolation of the recurrence vector segments supplied by means of "inherent distortions" or "outward factors" into certain number of sub-companies. This manner brings approximately isolating little varieties in a picture through and large in excessive recurrence sub-band photographs. consequently discrete wavelet trade (DWT) is a fine and appropriate method to be utilized as an arrangement framework author.

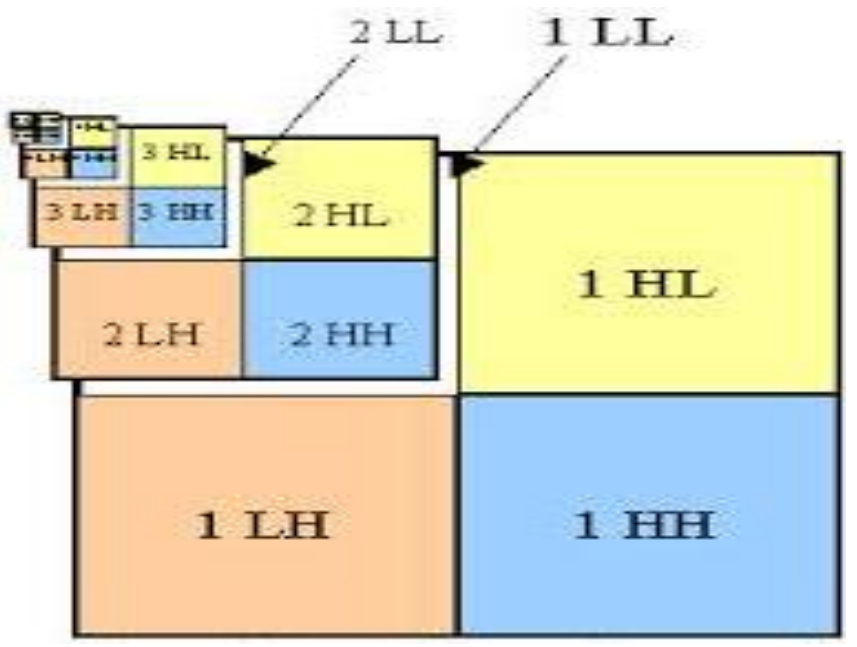

Fig. 3. Wavelet Decomposition levels

The 2 dimensional discrete wavelet decomposition of an finger print photograph effects in four decomposed sub-band fingerprint stages of photographs that are referred to as low-low frequency (LL), excessive-low frequency (HL), low-excessive frequency (LH) and high-high frequency $(\mathrm{HH})$. each of these sub-bands denotes unique finger print image residences. typically, the great and most of the strength vector are inside the low frequencies of pics and hence decomposition is commonly repeated at the low frequencies of snap shots LL sub-band handiest (dyadic decomposition). for example ' $n$ ' degree finger print picture decomposition, there are (three*n) +1 sub-bands to be had

$$
E_{k=\frac{1}{U V}} \sum_{x=1}^{U} \sum_{y=1}^{V}\left|K_{n}(x, y)\right|
$$

The recurrence vitality of all the sub-band coefficients are utilized separately as highlight vectors which are called sub-band recurrence vitality vectors ( ). The sub-band vitality vectors are determined utilizing the above condition (1). Each unique mark picture experiences limit of six-level deterioration after pre-preparing as appeared in Fig. 3. Since

\section{B.DWT FEATURE VECTOR EXTRACTION:}




\section{A Strange Algorithm For Fingerprint Based Age Estimation Using Frequency Domain And Pattern Recognition Techniques}

six level deterioration is best degree of getting recurrence vitality vectors. At each level, we get sub-groups and their recurrence vitality is determined. We get a sum of 19 sub-groups and the vitality vector will be $1 \times 19$ vectors toward the finish of the six-level disintegration for each unique mark picture. All fingerprints in the database experience the wavelet disintegration and the vitality vectors of the considerable number of pictures are put away.

\section{ICA FEATURE VECTOR EXTRACTION:}

Free Component Analysis (ICA) calculations are ordinarily utilized for example distinguishing proof procedures. It is a period differing marvels and computational procedure for discovering fundamental elements or segments from a multivariate measurable information vitality signal (multi dimensional sign) into added substance subcomponents unique finger impression picture. This procedure should be possible by expecting that the vitality subcomponents are non-Gaussian sign and that they are measurably autonomous from one to one another. All in all, Independent Component Analysis ICA can't distinguish the genuine number of source signals; only right collecting of neither the source signals, nor the best possible scaling (counting sign) of the source signals.ICA is an exceptional instance of visually impaired source detachment. A typical model for ICA strategy is the "mixed drink party issue" of tuning in to one individual's discourse in an uproarious zone.

\section{D.COMBINED FEATURE VECTOR:}

In the wake of experiencing the procedure two dimensional Discrete Wavelet Transform and Independent Component Analysis vitality include vector extraction, the vitality highlight vectors are put away independently. The accompanying advance is to consolidate both the recurrence vitality highlight vectors and time vitality vectors into a solitary vitality vector that stores the recurrence area and spatial space data of a unique mark. The 19 highlight vector from the DWT and the 512 element vector from ICA are consolidated to shape 1 x 531 vitality include vectors for a solitary unique finger impression picture. For every one of the fingerprints in the database, this methodology is pursued as the equivalent and a database include vector is made. This database include vector contains all the element vectors of the unique finger impression pictures in the database. In the event that there are - k-fingerprints in the database, at that point the size of the database vitality highlight vector will be $\mathrm{kx}$ 531.A chart was plotted in the wake of finding the joined element vector. Till date spatial highlights were utilized for examination and are settled. We propose this framework for the utilization of recurrence area examination to gain a person's age.

\section{E. CLASSIFIER:}

The qualities from joined vector are as marks. Multiclass SVM method is utilized as a classifier. It characterizes diverse age bunches by doling out various marks by utilizing bolster vector machine, where the names are drawn from a foreordained arrangement of a few components. Grouping of pictures can be practiced utilizing SVMs. Conditional outcomes demonstrate that SVMs accomplish significantly higher pursuit precision than conventional question refinement plans.

\section{ESTIMATION OF AGE}

Every one of the fingerprints utilized for our calculation was taken continuous broadly from the individuals having a place with various ages 10-20, 21-30, 31-40, 41-50 and 51-60 separately and ethnic gatherings. Every one of these fingerprints are optical examined pictures. The fingerprints of various age gathering are assembled and kept as the database fingerprints for preparing. When the ideal database is shaped every one of the fingerprints in it experiences the component vector extraction as clarified in the past segments.

\section{STEPS INVOLVED IN ESTIMATING THE AGE OF AN INDIVIDUAL USING THE QUERY FINGERPRINT:}

(i) Resize the unique mark to $512 \times 512$ by experiencing Pre-handling stage.

(ii) Generate 19 recurrence vitality include vectors by decaying the pre-prepared picture in Discrete Wavelet Transform DWT process.

(iii) The pre-prepared picture likewise experiences in Independent Component Analysis ICA strategy to produce 512 time vitality highlight vectors.

(iv) Combine both the vitality include vectors to acquire $531(19+512=531)$ highlight vectors for the given inquiry unique mark picture.

(v) The acquired vitality include vector experiences Multi-class SVM classifier and it further experiences the estimation of Age to at last presentation the yield (Age).

\section{ESTIMATION OF AGE:}

The gathered fingerprints from unique mark scanner are assembled based on the ages. Various gatherings are shaped for preparing dependent on ages. The five age bunches that we have made for our test are 10-20, 21-30, 31-40, 41-50 and 51-60 age gatherings individually. These fingerprints are made to experience the recurrence vitality include vector extraction and example vitality highlight vector systems. Every one of the means continue as before for the unique mark pictures other than the adjustment in the gatherings of the fingerprints. The inquiry unique mark additionally experiences the recurrence vitality include vector extraction forms and the element vectors are acquired. The component vectors of the inquiry unique finger impression are presently prepared to experience the Multi-class SVM classifier[12] which is set up for the characterization reason. After the extraction, the arrangement is finished by methods for the Multi-class SVM strategy. The question vector is contrasted and all the five classes of ages and the class which structures close-by connection with the given preparing fingerprints is taken for the age gathering of the Query Print. 


\section{IV.PERFORMANCE ANALYSIS \& RESULTS}

The calculation was written in MATLAB 7.14 form R2012a and was kept running on Pentium Dual Core processor $2.00 \mathrm{GHz}$ with 40 GB Hard Disk memory with least of 2GB RAM. We have utilized 250 fingerprints for preparing and the rest were utilized for testing reason. The consequence of the age estimation procedure is appeared in Fig.4.

Results demonstrate to us that recurrence area examination of fingerprints can be followed in future to contemplate dermatoglyphics. The general achievement rate in age estimation is around $81.2 \%$ for our database. The diagram of the general achievement rate is appeared in Fig.

5.

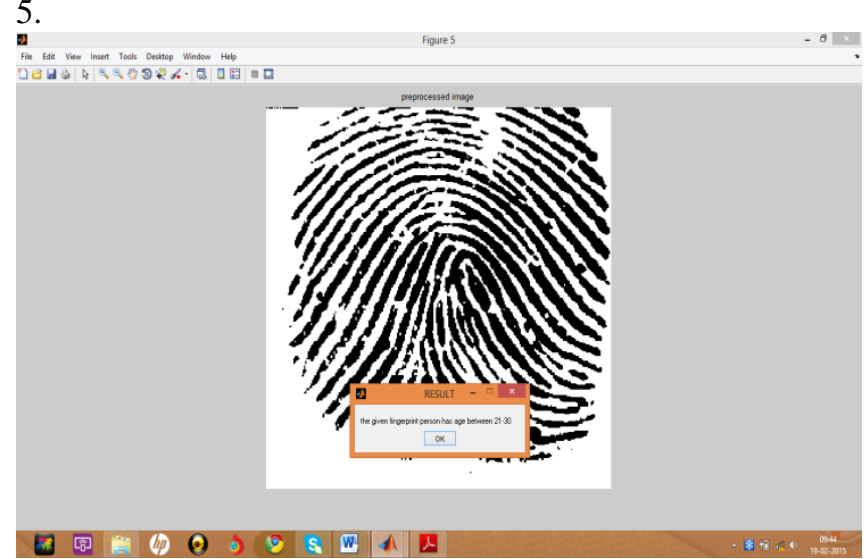

Fig. 4. Result of Age estimation

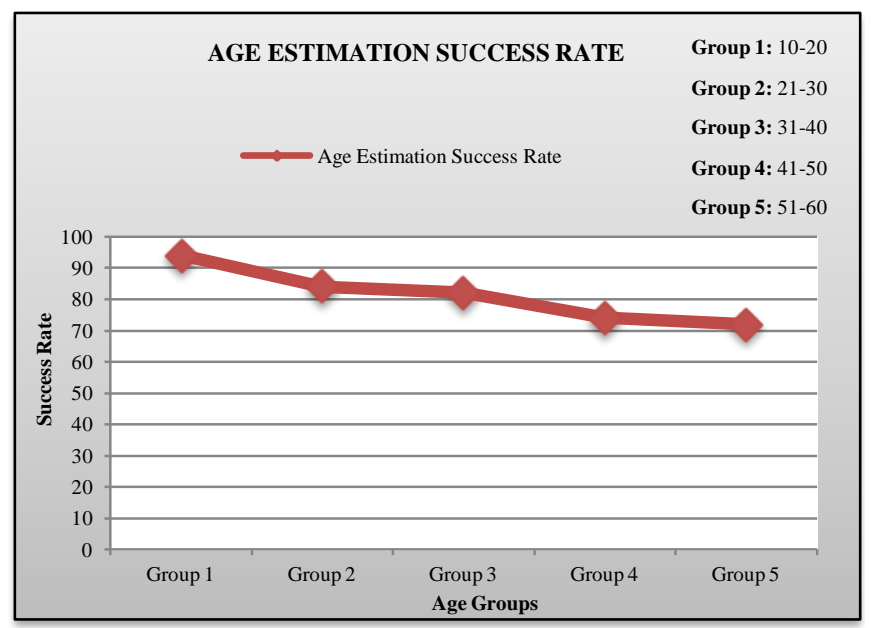

Fig. 5. Overall Success Rate of Age Estimation

The algorithm was employed only on optical scanned fingerprint images. It has been located that changing the database amount and excellent likewise improves the estimation method. the quantity of database fingerprints is a sizable paradigm. Trial consequences show a cover between the distinctive age gatherings fingerprints for low quality photographs. the dimensions of the Fingerprint image is predicated upon the development of the human frame. human beings of littler age bunch with a more body structure than their everyday development have fingerprints greater than their age collecting. those sorts of humans may additionally likewise have practicable consequences for age protecting. numerous age gatherings of fingerprints pix alternate in size and examples and thickness of edges and valleys.

\section{DESTINY WORKS}

The calculation turned into applied uniquely on optical filtered particular mark pix. it has been determined that converting the database amount and first-rate likewise improves the estimation process. the amount of database fingerprints is a enormous rule. Exploratory outcomes demonstrate a cowl between the various age gatherings fingerprints for low excellent images. the size of the Fingerprint photo is predicated upon the development of the human frame. people of littler age bunch with a greater body structure than their ordinary improvement have fingerprints more than their age gathering. those styles of people might also likewise have capability results for age masking. various age gatherings of fingerprints photographs range in size and examples and thickness of edges and valleys..

\section{REFERENCES}

1. P.Gnanasivam and Dr. S. Muttan, "Estimation of Age through Fingerprints using Wavelet remodel and Singular value Decomposition", global journal of Biometrics and Bioinformatics (IJBB), extent (6): difficulty (2):pp fifty eight-sixty seven. 2012.

2. Ravi Wadhwa and Maninder Kaur, "Age and Gender dedication from Finger Prints utilizing RVA and DCT Coefficients", IOSR journal of Engineering (IOSRJEN) e-ISSN: 2250-3021, P-ISSN 2278-8719 Vol. 3, difficulty 8 PP 05-09.

3. AapoHyvarinen and ErkkiOja, "free factor analysis: Algorithms and programs", Neural Networks studies center, Helsinki college of era, Finland, Neural Networks,13(four-5):411-430, 2000.

4. http://www.csie.ntu.edu.tw/ cjlin/papers/multisvm.pdf

5. Dipalee Gupta and Siddhartha Choubey, " Discrete Wavelet remodel for image Processing", global journal of rising generation and advanced Engineering, (ISSN 2250-2459, ISO 9001:2008 certified journal, extent four, trouble 3, March 2015)

6. Gheorghe Popa, RomicăPotorac and NicolaePreda, "method for fingerprints age assurance", Rom J Leg Med [2] 149 - 154 [2010], DOI: 10.4323/rjlm.2010.14.

7. D. Maltoni, D. Maio, A. okay. Jain, and S. Prabhakar, "guide of Fingerprint popularity", first ed., Springer, big apple, 2003.

8. Zain S. Barham and Dr.Allam Mousa, "unique mark recognition using MATLAB", http://eng.najah.edu/locales/eng.najah.edu/files/fingerprintrecog nition.pdf

9. Shimon okay. Modi, Prof. Stephen J. Elliott, Jeff Whetsone and Prof. Hakil Kim", effect of Age companies on Fingerprint recognition performance", IEEE Workshop on automatic Recognizable proof advanced technologies, 2007pp. 19-23.

10. Nathan C. Sickler and Stephen J Elliott, "An evaluation Of Fingerprint photo first-class across An aged populace Vis-A-Vis A 18-25 yr vintage population",IEEE-2005.

11. E.O. Omidiora, O. Ojo, N.A. Yekini and T.O. Tubi, "investigation, shape and execution of human particular mark designs framework "towards age and sexual orientation guarantee, edge thickness to valley thickness percentage (RTVTR) and side rely upon sex recognition", global magazine of advanced research in synthetic Intelligence, Vol. 1, No. 2, pp 57-63, 2012.

12. Ramesh.G.P A. Rajan," Glaucoma Detection in Optical Coherence Tomography photographs using Undecimated Wavelet transform.", research magazine of Pharmaceutical organic and Chemical Sciences, quantity 7, Issue3, Pages $878-885$. 